\title{
Creating Well-Defined Hot Spots for Surface-Enhanced Raman Scattering by Single-Crystalline Noble Metal Nanowire Pairs
}

\author{
Taejoon Kang, ${ }^{\dagger}$ Ilsun Yoon, ${ }^{\dagger}$ Ki-Seok Jeon, ${ }^{\S}$ Wonjun Choi, ${ }^{\ddagger}$ Yonghoon Lee, ${ }^{\|}$Kwanyong Seo, ${ }^{\dagger}$ \\ Youngdong Yoo, ${ }^{\dagger}$ Q-Han Park, ${ }^{+}$Hyotcherl Ihee, ${ }^{\dagger}$ Yung Doug Suh, ${ }^{*,}$ and Bongsoo Kim $*, \dagger$ \\ Department of Chemistry, KAIST, Daejeon 305-701, Korea, Division of Advanced Chemical Materials, KRICT, \\ Daejeon 305-600, Korea, Department of Physics, Korea University, Seoul 136-701, Korea, and Advanced \\ Photonics Research Institute, Gwangju Institute of Science and Technology, Gwangju 500-712, Korea
}

Received: October 23, 2008; Revised Manuscript Received: March 18, 2009

\begin{abstract}
Well-defined surface-enhanced Raman scattering (SERS) active systems were fabricated by single-crystalline noble metal nanowires. Crossed and parallel nanowire pairs were constructed by using a nanomanipulator to create SERS hot spots in the form of nanowire junction. SERS spectra of brilliant cresyl blue (BCB), $p$-mercaptoaniline ( $p \mathrm{MA})$, and $p$-mercaptobenzoic acid ( $p \mathrm{MBA}$ ) were observed at the junction of two nanowires. The SERS enhancement and polarization dependence are correlated well with the enhanced electric field intensities calculated by the finite difference time domain (FDTD) method for specific nanowire geometries. These simple and effective SERS active systems have a practical advantage that the hot spots can be readily located and visualized by an optical microscope. These well-defined SERS active systems based on noble metal nanowires can be further developed to find applications in a variety of biological and chemical sensing.
\end{abstract}

\section{Introduction}

Optimal molecular sensors would be characterized by high sensitivity, high selectivity, reproducibility, and reliable calibration. In terms of high selectivity, sensors based on optical detection offer advantages over other sensing methods because optical spectra can provide fingerprints for analytes. ${ }^{1,2}$ Among various detection methods, SERS stands out with its high sensitivity for single molecule detection due to intense electromagnetic fields which are associated with strong localized surface plasmon resonances of metallic nanostructures generated at hot spots. ${ }^{3-6}$ Because of these advantages of SERS, it can be applied to noninvasive chemical detection and identification of biological molecules such as DNAs and proteins. ${ }^{7-10}$

The strong enhancement of Raman signal has been attributed to "hot spots" that can be generated at the junction between metal surfaces upon irradiation with light. ${ }^{11-13}$ Since the SERS intensity is primarily dominated by these hot spots, much of the effort to develop SERS into an effective analytical tool has been devoted to fabricate well-defined, reproducible, and controlled hot spot structures. A number of promising structures have been suggested including the noble metal thin films, ${ }^{14,15}$ lithographically fabricated surfaces, ${ }^{16-18}$ nanoparticle dimers, ${ }^{19-22}$ aggregates, ${ }^{23,24}$ and nanowire bundles. ${ }^{25,26}$ At the atomic level, however, they still contain inhomogeneities of the surface structure, i.e., they are not well-defined yet on the atomic scale. ${ }^{27}$ In contrast to the past SERS experiments, we employed singlecrystalline noble metal nanowires that have atomically smooth surfaces $^{28}$ as building blocks for an optimal SERS active system.

Herein, we carried out a systematic study of noble metal nanowire pairs in order to construct a well-defined "metal/ molecule/metal" structure that could increase the SERS activity

\footnotetext{
* To whom correspondence should be addressed. Fax: +82-42-350-2810 E-mail: bongsoo@kaist.ac.kr and ydsuh@krict.re.kr.

${ }^{\dagger}$ KAIST.

$\S$ KRICT.

* Korea University.

"GIST.
}

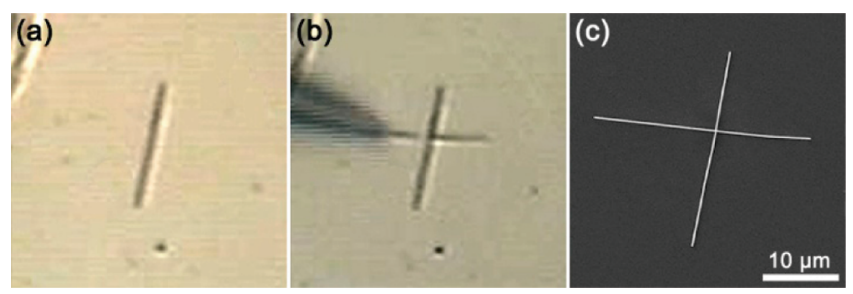

Figure 1. (a, b) Optical microscope images of the fabrication procedure of crossed Ag nanowires by using a nanomanipulator. (c) SEM image of the same crossed Ag nanowires.

to a very high extent. The crossed and parallel nanowire systems were fabricated by using a nanomanipulator with high reproducibility. More importantly, the SERS hot spots can be readily monitored by an optical microscope, thus most effective SERS measurement can be achieved with high efficiency. Strong SERS enhancement observed at the junction of two nanowires directly visualizes SERS hot spots. The observed SERS intensities and polarization dependence are correlated well with the electromagnetic field intensities calculated by an FDTD method for the specific nanowire geometries.

\section{Experimental Section}

Synthesis of Ag and Au Nanowires. Free-standing singlecrystalline Ag nanowires were synthesized by the vapor phase method that was described previously. ${ }^{28}$ In a typical synthesis, $0.2 \mathrm{~g}$ of $\mathrm{Ag}_{2} \mathrm{O}$ precursor powder (99.996\%, Sigma-Aldrich) was placed in an alumina boat at the middle of a 1 in. diameter horizontal quartz tube furnace. The Ag nanowires were grown about 3-4 cm downstream from the precursor on a Si substrate. The precursor vapor at a higher temperature zone $\left(T_{1}=\right.$ $900-1000{ }^{\circ} \mathrm{C}$ ) was carried downstream by the flow of $500 \mathrm{sccm}$ of argon gas at a pressure of 5 to 10 Torr to a lower temperature zone $\left(T_{2}=500{ }^{\circ} \mathrm{C}\right)$, where it grows to $\mathrm{Ag}$ nanowires.

Au nanowires were synthesized by direct evaporation of pure Au powder (99.99\%, Sigma-Aldrich), using the same horizontal 

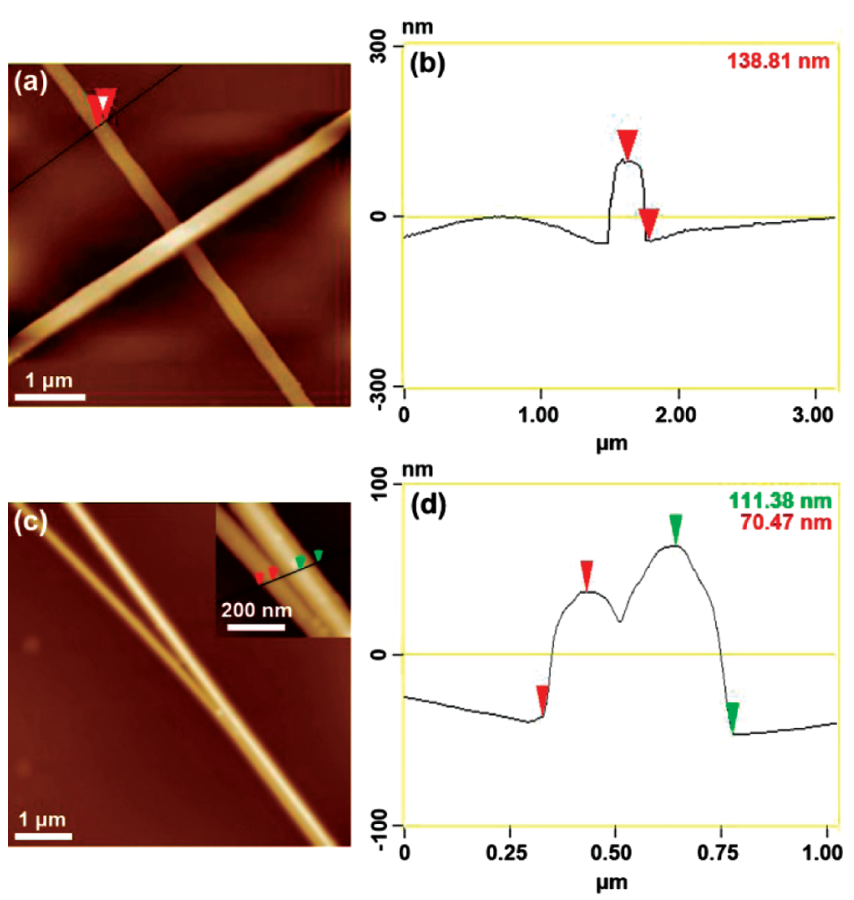

Figure 2. AFM images and section analyses of ( $a, b)$ crossed and (c, d) parallel Ag nanowires on which we obtained SERS spectra.

furnace system. ${ }^{29}$ The Au nanowires were grown on a sapphire substrate that was placed a few centimeters downstream from an alumina boat filled with $0.02 \mathrm{~g}$ of Au powder. Argon gas flowed at a rate of $100 \mathrm{sccm}$, maintaining the total pressure at 1 to 5 Torr. The higher temperature zone of the furnace was heated to $1100{ }^{\circ} \mathrm{C}$. The reaction time was about $30 \mathrm{~min}$.

Preparation of SERS Active Nanowire Pairs. The crossed and parallel Ag nanowire pairs were fabricated by transferring the as-synthesized $\mathrm{Ag}$ nanowires one by one onto a glass substrate by using a custom-built nanomanipulator. The nanomanipulator consists of a tungsten tip $(\sim 100 \mathrm{~nm}$ diameter at the end) mounted on a three-dimensional piezoelectric stage. Ten microliters of a $10^{-4} \mathrm{M}$ ethanolic solution of BCB (SignamAldrich) was dropped and spin coated (5000 rpm, $1 \mathrm{~min}$ ) onto nanowire pairs on the glass substrate. For Au nanowire pairs, as-grown Au nanowires were incubated first in a $3 \mathrm{mM}$ ethanolic solution of $p \mathrm{MA}$ (Sigma-Aldrich) or $p \mathrm{MBA}$ (Sigma-Aldrich) for $24 \mathrm{~h}$ and rinsed with an excess of ethanol. The crossed and parallel $\mathrm{Au}$ nanowire pairs were then constructed on a glass substrate by using the nanomanipulator.

Instrumentation. The atomic force microscope (AFM) images of crossed and parallel Ag nanowires were obtained with a Veeco bioscope AFM, using a Nanoscope IV controller that is mounted on a micromechanical stage on an inverted optical microscope (Axiovert 200, Zeiss). The scanning electron microscope (SEM) images of the Au nanowires were taken on a Phillips XL30S operated at $10 \mathrm{kV}$.

The Raman signals were obtained by two different custombuilt Raman systems. SERS spectra by Ag nanowires were measured with an AFM/confocal microscope. The $514.5 \mathrm{~nm}$ line of an argon-ion laser (Melles Griot) coupled into a single mode optical fiber was used as an excitation source. The laser beam size was expanded to a diameter of about $6 \mathrm{~mm}$ by using a telescope and inserted into a microscope entrance port. A dichroic mirror directs the excitation laser beam through an oil immersion microscope objective $(\times 50, \times 100$, Zeiss $)$ that focuses the beam upon the upper surface of a glass substrate. The SERS signal was collected with the same objective and recorded with
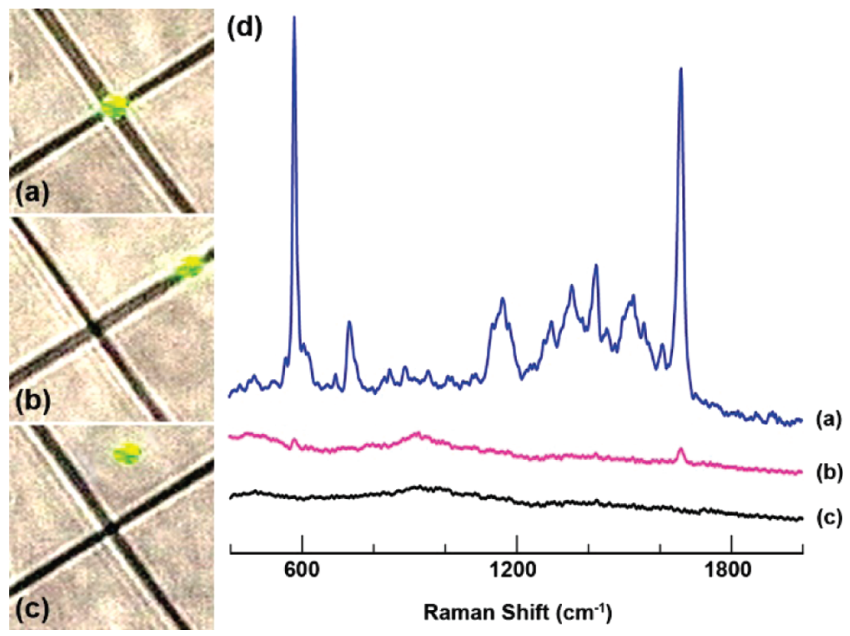

Figure 3. $(\mathrm{a}-\mathrm{c})$ Optical microscope images of crossed Ag nanowires. The green laser spot was focused on the crossing point of Ag nanowires, a single Ag nanowire, and a glass substrate, respectively. (d) The corresponding SERS spectra of BCB at the laser spots in panels a-c.

a liquid nitrogen cooled charge coupled device (CCD, Roper Scientific) mounted on the exit port of a monochromator (Holospec, Kaiser Optical Systems Inc.).

SERS spectra by Au nanowires were obtained with a microRaman system based on an Olympus BX41 microscope. The $633 \mathrm{~nm}$ radiation of a $\mathrm{He}-\mathrm{Ne}$ laser (Melles Griot) was used as an excitation source and the laser light was focused on a sample through a $\times 100$ objective (Mitutoyo). The SERS signal was recorded with a thermoelectrically cooled electron multiplying charge coupled device (EMCCD, Andor) mounted on the spectrometer with a 1200 groove/mm grating. A holographic notch filter was used to reject the laser light.

\section{Results and Discussion}

Single-crystalline noble metal nanowires synthesized by the vapor phase method have clean surfaces that can be used to build well-defined SERS active systems. To fabricate a welldefined system for SERS using these nanowires, we crossed two nanowires and constructed a plasmonic hot site at the junction (Figure 1). The nanowires were transferred onto a glass substrate by using the nanomanipulator. Figure 1a,b shows the fabrication procedure of crossed Ag nanowires. The nanomanipulator picked up a single free-standing nanowire by softly touching it and transferred the nanowire to the substrate (Figure 1a) and transferred another nanowire to complete the crossed nanowire architecture (Figure 1b). A plasmonic gap consisting of two nanowires was constructed. The optical microscope image of the tungsten tip of the nanomanipulator and nanowires is seen in Figure 1b. Figure 1c shows a SEM image of the same crossed nanowires. The parallel Ag nanowire structure was also fabricated by a similar procedure. Since the nanowires had been monitored conveniently through an optical microscope, fabricating nanowire pairs and locating the junction position were easily accomplished.

The AFM images and cross section analyses of the crossed and parallel Ag nanowires are shown in Figure 2. Since the SERS spectra by Ag nanowires were acquired by using a custom-built confocal microscope that also has an AFM setup, the AFM images of the Ag nanowires were obtained right after the SERS measurement. The AFM (Bioscope, Digital Instruments Inc., Veeco Metrology Group) using a Nanoscope IV 

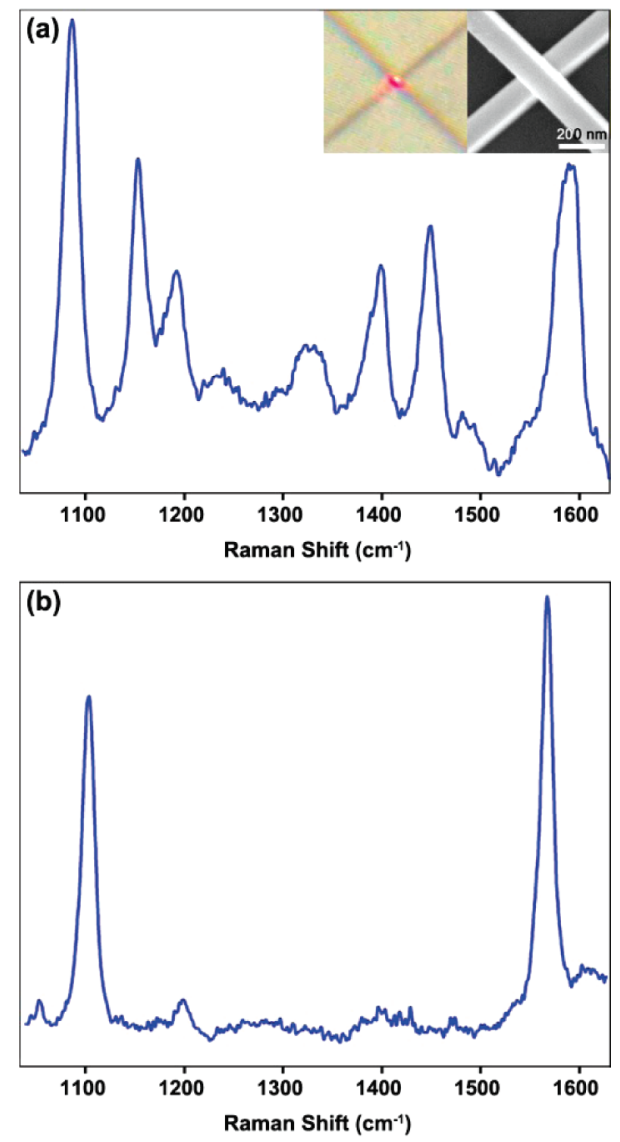

Figure 4. SERS spectra of (a) $p \mathrm{MA}$ and (b) $p \mathrm{MBA}$ for the crossed $\mathrm{Au}$ nanowires. The insets are an optical microscope image and a magnified SEM image of crossed Au nanowires.

controller is mounted on a micromechanical stage on an inverted optical microscope (Axiovert 200, Zeiss).

It is clear in micrographs that the crossed and parallel nanowires were made by two single nanowires and not by a bundle. Moreover, SERS hot spots are directly visualized at the junction of two nanowires. The inset of Figure $2 \mathrm{c}$ also shows that the distance between two nanowires is sufficiently small to create SERS hot spots.

Optical microscope images of crossed $\mathrm{Ag}$ nanowires are shown in Figure $3 \mathrm{a}-\mathrm{c}$. Ten microliters of a $10^{-4} \mathrm{M}$ ethanolic solution of BCB was dispersed on this system by spin coating. Next, $150 \mu \mathrm{W}$ of $514.5 \mathrm{~nm}$ argon-ion laser radiation was used to excite samples with a data collection time of $30 \mathrm{~s}$. The green laser spot is seen on the crossing point of two nanowires (Figure $3 a$ ), a single nanowire (Figure 3b), and a glass substrate (Figure $3 c)$, respectively. The corresponding SERS spectra of BCB are displayed in Figure 3d. The strong Raman bands at 580 and $1650 \mathrm{~cm}^{-1}$ have been assigned to aromatic $\mathrm{C}-\mathrm{C}$ bending and $\mathrm{C}=\mathrm{C}$ stretching modes, respectively. ${ }^{30}$ The intensities of peak at 580 and $1650 \mathrm{~cm}^{-1}$ increase by about 40 times and many other peaks around $1400 \mathrm{~cm}^{-1}$ appear when the laser focusing point is moved from a single nanowire (spectrum $b$ of Figure $3 d$ ) to the crossing point of two nanowires (spectrum a of Figure 3d). This indicates that the SERS signal is strongly enhanced for the $\mathrm{BCB}$ molecules adsorbed onto the hot spots at the junction of two nanowires.

We have estimated the enhancement factor $(\mathrm{EF})$ by comparing the peak intensities of SERS for crossed $\mathrm{Ag}$ nanowires and those for a single nanowire in Figure 3. The EF was calculated according to the following expression: ${ }^{31}$

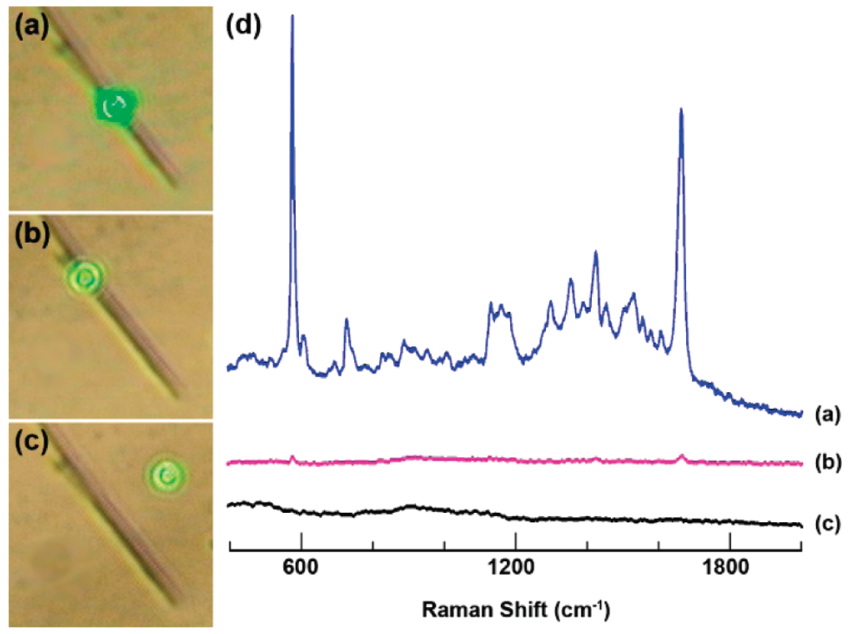

Figure 5. $(a-c)$ Optical microscope images of parallel Ag nanowires. The green laser spot was focused on the parallelly touching Ag nanowires, separated Ag nanowires, and a glass substrate, respectively. (d) The corresponding SERS spectra of BCB at the laser spots in panels $\mathrm{a}-\mathrm{c}$.

$$
\mathrm{EF}=\left[I_{\text {cross }}\right] /\left[I_{\text {single }}\right] \times\left[N_{\text {single }}\right] /\left[N_{\text {cross }}\right]
$$

where $N_{\text {single }}$ is the number of molecules contributing to the observed SERS signal for a single nanowire and is proportional to the laser-illuminated area. $N_{\text {cross }}$ is the number of molecules at the crossing point and is proportional to the effective crossing area, not to the laser-illuminated area, which is much larger than the effective crossing area. Thus $N_{\text {single }}$ is much greater than $N_{\text {cross. }} I_{\text {cross }}$ and $I_{\text {single }}$ are intensities of the observed SERS spectra. Assuming uniform adsorption, we get

$$
\mathrm{EF}=\left[I_{\text {cross }}\right] /\left[I_{\text {single }}\right] \times\left[A_{\text {single }}\right] /\left[A_{\text {cross }}\right]
$$

The $A_{\text {single }}$ indicates the laser-illuminated area of a single nanowire and $A_{\text {cross }}$ is the surface area of the hot spot region. The nanowire diameter is about $140 \mathrm{~nm}$ (Figure 2a). The $A_{\text {cross }}$ for which the gap distance is less than $5 \mathrm{~nm}$ is about $2800 \mathrm{~nm}^{2}$. For the single nanowire, $A_{\text {single }}$ is about $2.2 \times 10^{5} \mathrm{~nm}^{2}$ when the laser spot diameter is $500 \mathrm{~nm}$. The intensity ratio of SERS from the crossing point and single nanowires is about 40 for the peak at $580 \mathrm{~cm}^{-1}$. Thus, the EF achieved by the crossed nanowires is about $3.1 \times 10^{3}$ compared to the SERS signal for a single nanowire. This enhancement can be attributed to increased local electric fields near the crossing point of nanowires due to the excitation of surface plasmon resonances.

SERS spectra from the crossing point of Au nanowires were also obtained with an excitation source of $633 \mathrm{~nm} \mathrm{He}-\mathrm{Ne}$ laser. Generally, gold is chemically inert and biocompatible compared to silver, which is oxidized readily in air and toxic to cells. ${ }^{32}$ The surface of $\mathrm{Au}$ nanowires can be modified by various molecules through a gold-sulfur atom chemical bond to optimize the surface activities. As-synthesized Au nanowires were incubated in an ethanolic solution of thiol molecules and rinsed with an excess of ethanol. SAM was formed and the thiol molecules were uniformly coated on nanowire surfaces. The nanowires were then transferred onto a substrate by using a nanomanipulator and crossed nanowires were constructed. The crossing gap of two nanowires generates a hot spot. Figure 4 shows the SERS spectra of $p$ MA and $p$ MBA from the crossed Au nanowires. A strong SERS signal is observed at the junction point of two crossed Au nanowires, as observed for the crossed Ag nanowires.

We constructed crossed nanowires with single-crystalline noble metal nanowires to provide stronger SERS signals than a 

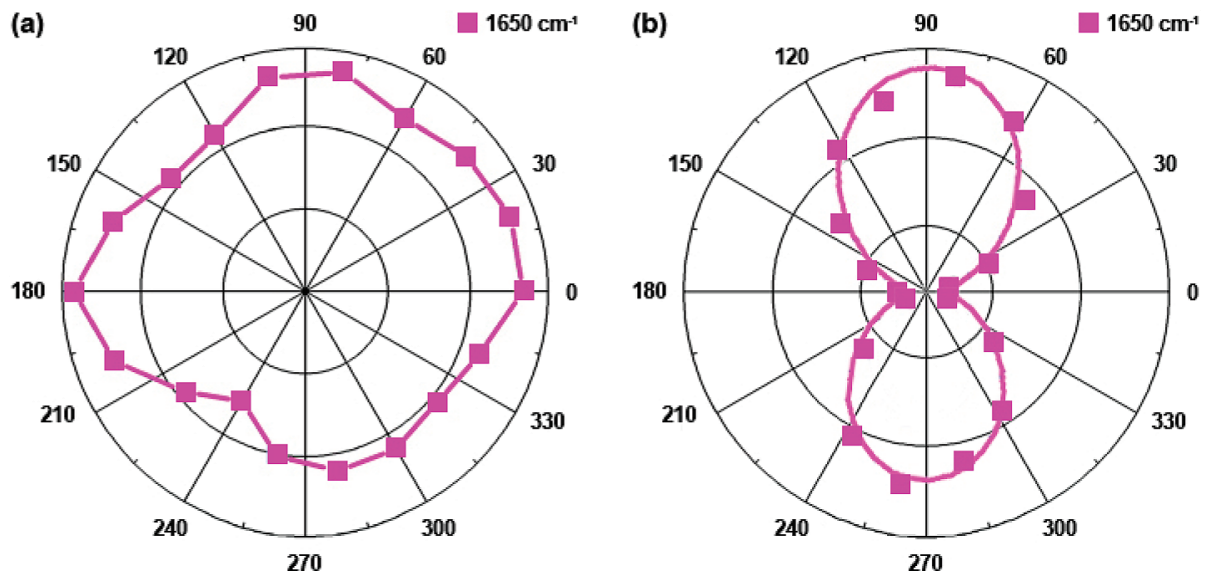

Figure 6. Polar plots of integrated SERS intensity of the $1650 \mathrm{~cm}^{-1}$ Raman band of BCB at (a) crossed Ag nanowires and (b) parallel Ag nanowires.

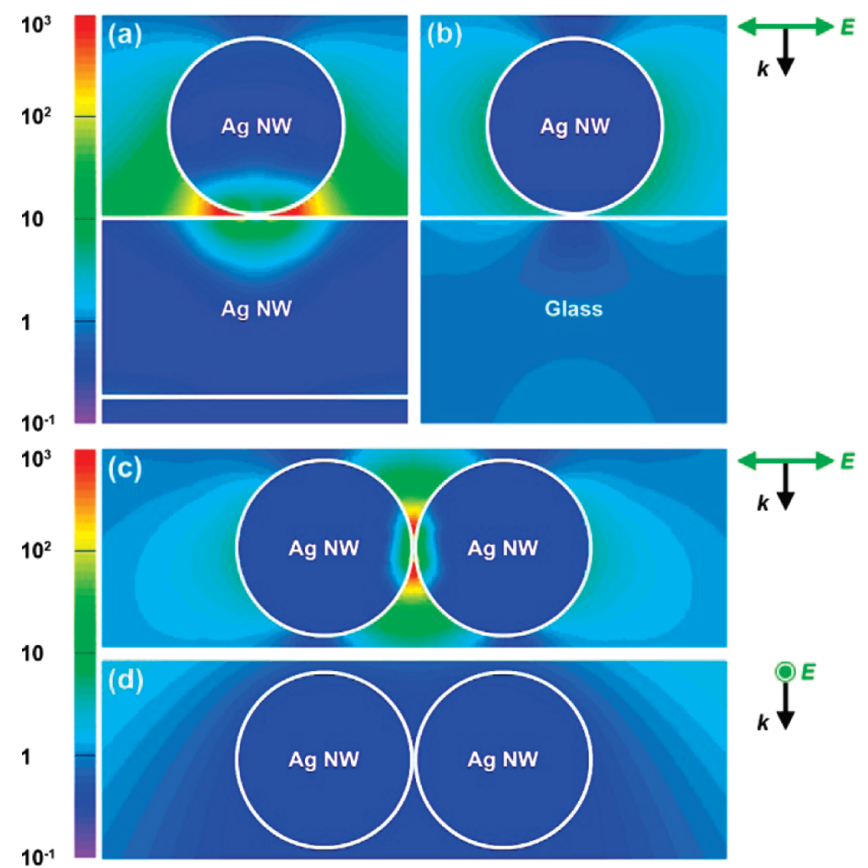

Figure 7. Local electric field intensities $|E|^{2}$ of (a) crossed $\mathrm{Ag}$ nanowires, (b) a single Ag nanowire on a glass substrate, and (c, d) parallel $\mathrm{Ag}$ nanowires with different incident polarization directions. The FDTD method was used for calculations with an incident light wavelength of $514.5 \mathrm{~nm}$.

single nanowire. The observed enhancement confirms that the electromagnetic fields are localized at the junction between two nanowires. With this SERS active system, it should be possible to engineer the hot spots with predictable electromagnetic responses.

Figure $5 \mathrm{a}-\mathrm{c}$ shows the optical images of parallel $\mathrm{Ag}$ nanowires through a $\times 50$ oil immersion objective. To investigate the influence of the interwire distance on the SERS signal, we first measured the SERS spectrum from parallelly touching nanowires (Figure 5a). We then moved the laser focusing point to separated nanowires (Figure 5b) and a glass substrate (Figure $5 \mathrm{c})$. The interwire distance in Figure $5 \mathrm{~b}$ is about $300 \mathrm{~nm}$ from the AFM analysis (Figure 2c). Figure 5d illustrates the corresponding SERS spectra of BCB. The SERS intensities of the parallel nanowires were strongly increased compared to those of the separate nanowires. When the interwire distance is sufficiently small, the collected Raman signal can be enhanced by pairwise electromagnetic interactions. ${ }^{33}$ These SERS spectra from molecules at the gap between two nanowires also suggest that the parallel nanowire junction can serve as a Raman hot spot.

The polarization dependences of crossed and parallel Ag nanowires are plotted as a function of $\theta$ for integrated SERS intensity of the $1650 \mathrm{~cm}^{-1}$ Raman band (Figure 6). $\theta$ is the angle between the nanowire axis and the polarization direction of light. For the crossed nanowires system, the polarization dependence shows 4-fold symmetry, as is expected from the geometry of the system. On the other hand, the parallel nanowires system shows 2-fold symmetry and $\cos ^{2} \theta$ dependence similarly to a single Ag nanowire. ${ }^{28}$ It should be noted that the SERS signal of parallel nanowires is much increased compared to that of a single nanowire. Yang's and Moskovits's groups observed similar anisotropic enhancement for a monolayer and a raft of Ag nanowires, respectively. ${ }^{33,34}$

To interpret the observed SERS results in terms of the electromagnetic enhancement mechanism of SERS, local electric field intensities $|E|^{2}$ of crossed $\mathrm{Ag}$ nanowires, a single $\mathrm{Ag}$ nanowire, and parallel Ag nanowires with different polarization directions of incident light were calculated by the fully 3-dimensional FDTD method at a wavelength of $514.5 \mathrm{~nm}$ (Figure 7). In the FDTD calculation, we applied Drude's metal model and the nanowires were excited by a Gaussian beam that has $500 \mathrm{~nm}$ full width at half-maximum (fwhm) and unit intensity. ${ }^{35}$ The diameters of the nanowires were set as $100 \mathrm{~nm}$. The $k$ - and $E$-vectors indicate the incident direction of laser light and the polarization direction, respectively. This calculation permits an estimation of the electromagnetic contribution to the SERS enhancement for various structures of nanowire pairs. The simulation shows that the local electric field is strongly enhanced near the contact point of two nanowires.

These calculated results also allow us to assess the polarization dependence of the SERS signal observed for parallel Ag nanowires. From the calculated electromagnetic fields of parallel nanowires at various incident light polarizations, we find that the highest fields are excited when the polarization direction is perpendicular to the long axis of nanowires, which agrees well with the observed polarization dependence of SERS intensities (Figure 6b).

The calculated values and experimental SERS intensities for all Ag nanowire geometries in Figure 7 are given in Table 1. For the parallel nanowires and a single nanowire, the integration of SERS intensities, $\int|E|^{4} \mathrm{~d} s$, was taken over the volume of a cylindrical shell covering the nanowire surface with $10 \mathrm{~nm}$ shell thickness and a length of $500 \mathrm{~nm}$. For the crossed nanowires 
TABLE 1: Results from FDTD Calculation Compared with Experimental SERS Intensity

\begin{tabular}{llcc}
\hline & \multicolumn{2}{c}{ FDTD simulation } & \\
\cline { 2 - 3 } nanowire geometry & $\max |E|^{2}$ & $\int|E|^{4} \mathrm{ds}$ & $\begin{array}{c}\text { SERS } \\
\text { intensity (exptl) }\end{array}$ \\
\hline crossed nanowires & 2096 & $5.8 \times 10^{9}$ & 40 \\
parallel nanowires $^{a}$ & 1031 & $7.0 \times 10^{9}$ & 50 \\
single nanowire $^{a}$ & 5 & $3.1 \times 10^{7}$ & 1
\end{tabular}

${ }^{a}$ The polarization direction of incident light is perpendicular to the long axis of nanowires.

system, the $\int|E|^{4} \mathrm{~d} s$ value was calculated by integrating inside the volume element of only the hot spot region characterized by a cube of $50 \mathrm{~nm}$ sides centered at the junction. The calculated $\int|E|^{4} \mathrm{~d} s$ values for the nanowire pair systems (crossed and parallel) were about 200 times larger than that of the single nanowire system, agreeing reasonably well with the experimental SERS intensity ratio.

In these nanowire pair systems, the electromagnetic field enhancement can be better defined and the adsorption of target molecules on the almost atomically smooth metal surfaces would be better organized than those on the conventional SERS systems. After further optimization including the uniformity control of the diameters of the nanowires, these systems would be able to contribute significantly to enhanced reproducibility of SERS sensors. As another merit, multiple SERS detectors, whose surfaces can be modified independently, could be fabricated into a biochip utilizing the small size of nanowire systems of the order of micrometers.

\section{Conclusion}

Well-defined SERS active systems are fabricated by two single-crystalline noble metal nanowires. The almost atomically flat surfaces of metallic nanowires provide a well-defined plasmonic nanocavity, creating SERS hot spots at a nanowire junction. The intense SERS enhancements observed at the junction are fully consistent with FDTD calculation results. These simple nanowire based structures offer the unique and practical advantage that the SERS hot spots can be readily located and visualized by an optical microscope. These systems can lead us to a deeper understanding of molecule-metal interaction at metal nanostructures as well as development of molecular electronic devices.

Acknowledgment. This research was supported by KOSEF through NRL (ROA-2007-000-20127-0), Center for Nanostructured Materials Technology under "21st Century Frontier R\&D Programs" of the MOST (06K1501-02620), and Nano R\&D program through the KOSEF, MEST (2008-02824).

Supporting Information Available: Experimental details including SERS spectra and FDTD calculations. This material is available free of charge via the Internet at http://pubs.acs.org.

\section{References and Notes}

(1) Liu, G. L.; Lu, Y.; Kim, J.; Doll, J. C.; Lee, L. P. Adv. Mater. 2005, 17, 2683.

(2) Prokes, S. M.; Glembocki, O. J.; Rendell, R. W.; Ancona, M. G. Appl. Phys. Lett. 2007, 90, 093105.

(3) Moskovits, M. Rev. Mod. Phys. 1985, 57, 783.

(4) Nie, S.; Emory, S. R. Science 1997, 275, 1102.

(5) Kneipp, K.; Kneipp, H.; Itzkan, I.; Dasari, R. R.; Feld, M. S. Chem. Rev. 1999, 99, 2957.

(6) Michaels, A. M.; Nirmal, M.; Brus, L. E. J. Am. Chem. Soc. 1999, 121,9932 .

(7) Braun, G.; Lee, S. J.; Dante, M.; Nguyen, T.-Q.; Moskovits, M.; Reich, N. J. Am. Chem. Soc. 2007, 129, 6378.

(8) Bonham, A. J.; Braun, G.; Pavel, I.; Moskovits, M.; Reich, N. O. J. Am. Chem. Soc. 2007, 129, 14572.

(9) Sun, L.; Song, Y.; Wang, Li; Guo, C.; Sun., Y.; Liu, Z.; Li, Z. J. Phys. Chem. C 2008, 112, 1415.

(10) Pavel, I.; McCarney, E.; Elkhaled, A.; Morrill, A.; Plaxco, K.; Moskovits, M. J. Phys. Chem. C 2008, 112, 4880.

(11) Michaels, A. M.; Jiang, J.; Brus, L. J. Phys. Chem. B 2000, 104, 11965.

(12) Braun, G.; Pavel, I.; Morrill, A. R.; Seferos, D. S.; Bazan, G. C.; Reich, N. O.; Moskovits, M. J. Am. Chem. Soc. 2007, 129, 7760.

(13) Lee, S. J.; Guan, Z.; Xu, H.; Moskovits, M. J. Phys. Chem. C 2008, 111,17985 .

(14) Goudonnet, J. P.; Bijeon, J. L.; Warmack, R. J.; Ferrell, T. L. Phys. Rev. B 1991, 43, 4605 .

(15) Yang, X. M.; Ajito, K.; Tryk, D. A.; Hashimoto, K.; Fujishima, A. J. Phys. Chem. 1996, 100, 7293.

(16) Tian, J.-H.; Liu, B.; Li, X.; Yang, Z.-L.; Ren, B.; Wu, S-T.; Tao, N.; Tian, Z.-Q. J. Am. Chem. Soc. 2006, 128, 14748.

(17) Ward, D. R.; Grady, N. K.; Levin, C. S.; Halas, N. J.; Wu, Y.; Nordlander, P.; Natelson, D. Nano Lett. 2007, 7, 1396.

(18) Zhang, X.; Zhao, J.; Whitney, A. V.; Elam, J. W.; Van Duyne, R. P. J. Am. Chem. Soc. 2006, 128, 10304.

(19) Suh, Y. D.; Schenter, G. K.; Zhu, L.; Lu, H. P. Ultramicroscopy 2003, 97,89

(20) Talley, C. E.; Jackson, J. B.; Oubre, C.; Grady, N. K.; Hollars, C. W.; Lane, S. M.; Huser, T. R.; Norlander, P.; Halas, N. J. Nano Lett. 2005, $5,1569$.

(21) Svedberg, F.; Li, Z.; Xu, H.; Käll, M. Nano Lett. 2006, 6, 2639.

(22) Sawai, Y.; Takimoto, B.; Nabika, H.; Ajito, K.; Murakoshi, K.

J. Am. Chem. Soc. 2007, 129, 1658.

(23) Kneipp, K.; Wang, Y.; Kneipp, H.; Perelman, L. T.; Itzkan, I.;

Dasari, R. R.; Feld, M. S. Phys. Rev. Lett. 1997, 78, 1667.

(24) Lu, Y.; Liu, G. L.; Lee, L. P. Nano. Lett. 2005, 5, 5.

(25) Tao, A.; Kim, F.; Hess, C.; Goldberger, J.; He, R.; Sun, Y.; Xia, Y.; Yang, P. Nano Lett. 2003, 3, 1229.

(26) Lee, S. J.; Morrill, A. R.; Moskovits, M. J. Am. Chem. Soc. 2006, $128,2200$.

(27) Tian, Z.-Q.; Ren, B.; Wu, D.-Y. J. Phys. Chem. B 2002, 106, 9463.

(28) Mohanty, P.; Yoon, I.; Kang, T.; Seo, K.; Varadwaj, K. S. K.; Choi, W.; Park, Q.-H.; Ahn, J. P.; Suh, Y. D.; Ihee, H.; Kim, B. J. Am. Chem. Soc. 2007, 129, 9576.

(29) Yoon, I.; Kang, T.; Choi, W.; Kim, J.; Yoo, Y.; Joo, S.-W.; Park, Q.-H.; Ihee, H.; Kim, B. J. Am. Chem. Soc. 2009, 132, 758.

(30) Vannier, C.; Yeo, B.-S.; Melanson, J.; Zenobi, R. Rev. Sci. Instrum. 2006, 77, 023104 .

(31) Nokoobakht, B.; Wang, J.; El-Sayed, M. A. Chem. Phys. Lett. 2002, $366,17$.

(32) Love, J. C.; Estroff, L. A.; Kriebel, J. K.; Nuzzo, R. G.; Whitesides, G. M. Chem. Rev. 2005, 105, 1103.

(33) Tao, A.; Yang, P. J. Phys. Chem. B 2005, 109, 15687.

(34) Jeong, D. H.; Zhang, Y.; Moskovits, M. J. Phys. Chem. B 2004, $108,12724$.

(35) Taflove, A.; Hagness, S. C. Computational Electrodynamics: The Finite-Difference Time-Domain Method, Bk\&Cd ed.; Artech House: Norwood, MA, 2000.

JP809391C 